\title{
On a multipoint nonlocal initial value problem for a singularly-perturbed first-order ODE
}

\author{
Dovlet M. Dovletov \\ Communicated by Sandra Pinelas
}

\begin{abstract}
A linear first order ordinary differential equation (ODE) with a positive parameter $\varepsilon$ and a multipoint nonlocal initial value condition (NLIVC) is considered. The existence of a classical solution of the multipoint nonlocal initial value problem (NLIVP) is proved. A uniform on $\varepsilon$ a priori estimate and asymptotic expansion of smooth solution is obtained. The differential problem with integral kind of NLIVC is considered and reduced to appropriate multipoint NLIVP.
\end{abstract}

Keywords. Ordinary differential equation, positive parameter, small parameter, multipoint problem, nonlocal initial value problem, nonlocal initial value condition, a uniform on parameter a priori estimate, an asymptotic expansion, integral kind of nonlocal initial value condition.

2010 Mathematics Subject Classification. 34B10.

\section{Introduction}

A large number of different works in the field of differential equations on a small parameter were strongly motivated by the classical article of A.N. Tikhonov [1]. General approach for an asymptotic expansion in terms of a small parameter was described by M.I. Vishik and L.A. Lyusternik in their outstanding joint paper [2].

A lot of works in the field of nonlocal boundary value problems were stimulated by the famous paper of A.V. Bitsadze and A.A. Samarskii [3]. The differential and difference interpretations of nonlocal boundary value problems were considered by V.A. Il'in and E.I. Moiseev [4-6] for the Sturm-Lioville operator and the Poisson's equation.

These fields of research remain relevant. For this reason our original paper deals with NLIVP for ODE with a positive parameter at the derivative. Actually, topical works by A.B. Vasil'eva, V.F. Butuzov, A. Ashyralyev, P.E. Sobolevskii, E.P. Doolan, J.J.H. Miller, W.H.A. Schilders [7-9] and other papers [10-12] influenced the author's investigation.

The approach of our original article is based on works [13-15] in general. We 
establish a uniform on a parameter a priori estimate for the classical solution of NLIVP and obtain an appropriate asymptotic expansion of the smooth solution in terms of a small parameter.

We consider a multipoint $\operatorname{NLIVP}^{1}$ with a parameter $\varepsilon>0$

$$
\varepsilon u^{\prime}(x)+a(x) u(x)=f(x), \quad x>0, u(0)-\sum_{k=1}^{n} \alpha_{k} u\left(\zeta_{k}\right)=\phi
$$

for $\zeta_{k} \in(0, X), X>0$ and $\alpha_{k} \in R, k=1, \cdots, n, \phi \in R$.

For the classical solution of the Cauchy problem

$$
\varepsilon u^{\prime}(x)+a(x) u(x)=f(x), \quad x>0, u(0)=\phi
$$

with smooth functions $f(x)$ and $a(x), a(x) \geq a>0, \forall x \geq 0$, the a priori estimate

$$
|u(x)| \leq C\left(|\phi|+\max _{0 \leq t \leq X}|f(t)|\right), \quad 0 \leq x \leq X
$$

is a uniform on $\varepsilon$ and independent on $x$ [9, p. 13-14].

In this paper, we establish a uniform on $\varepsilon$ a priori estimate for the smooth solution of NLIVP. The NLIVP is considered under specific conditions concerning the values and signs of the corresponding coefficients $\alpha_{k}, k=1, \cdots, n$ and the layout of the nonlocal points $\zeta_{k}, k=1, \cdots, n$. Generally, NLIVP with $\alpha_{k}, k=1, \cdots, n$ having the same sign is considered in Section 2. The corresponding NLIVP with $\alpha_{k}, k=1, \cdots, n$ having different signs is considered in Section 3. An integral kind of NLIVC is considered in Section 4. Finally, an appropriate asymptotic expansion of the solution in terms of a small parameter is considered in Section 5 .

\section{Multipoint problem with NLIVC having the same sign factors}

\section{Consider NLIVP}

$$
\begin{gathered}
L u \equiv \varepsilon u^{\prime}(x)+a(x) u(x)=f(x), \quad x>0, \\
\ell u \equiv u(0)-\sum_{k=1}^{n} \alpha_{k} u\left(\zeta_{k}\right)=\phi
\end{gathered}
$$

for smooth functions $f(x)$ and $a(x), a(x) \geq a>0$ for $x \geq 0, \varepsilon>0$, $\alpha_{k} \neq 0$ for $k=1, \cdots, n$ and $0<\zeta_{1}<\zeta_{2}<\cdots<\zeta_{n}<X, \quad X \in R, \phi \in R$.

Definition 2.1. A parameter value $\varepsilon^{*}$ is called a critical parameter ${ }^{2}$, if NLIVP

\footnotetext{
${ }^{1}$ We use the term "nonlocal initial value problem" for considering in this paper problems. By this we follow $[4,5]$ where the term "first" and "second kind" were introduced for NLBVP's.

${ }^{2}$ Here and throughout the paper, the symbol $\varepsilon^{*}$ denotes the critical parameter by default.
} 
(1), (2) has not the unique solution for $\varepsilon=\varepsilon^{*}$.

Lemma 2.2. The classical solution of NLIVP (1), (2) exists for some value of $\varepsilon$ if and only if $\ell \omega(\varepsilon) \neq 0$ for $\ell$ defined by (2) and $\omega(x)=\exp \left(-\varepsilon^{-1} \int_{0}^{x} a(t) d t\right)$.

Proof. Using the variation of constants method, we have a formal representation for the solution of the NLIVP (1), (2)

$$
u(x)=\omega(x)\left[C+\varepsilon^{-1} \int_{0}^{x} \omega^{-1}(\tau) f(\tau) d \tau\right]
$$

with

$$
C=[\ell \omega(\varepsilon)]^{-1}\left[\phi+\varepsilon^{-1} \sum_{k=1}^{n} \alpha_{k} \omega\left(\zeta_{k}\right) \int_{0}^{\zeta_{k}} \omega^{-1}(\tau) f(\tau) d \tau\right] .
$$

Therefore, the constant $C$ is uniquely determined if and only if $\ell \omega(\varepsilon) \neq 0$ because if $\ell \omega(\varepsilon)=0$, then $C$ is undefinable or equal to an arbitrary real number alternatively. Lemma 2.2 is proved.

Lemma 2.3. If $\alpha_{k}, k=1, \cdots, n$ have arbitrary signs and if $\sum_{k=1}^{n} \alpha_{k}>1$ for NLIVP (1), (2), then a critical parameter exists.

Proof. Actually, $\ell \omega(\varepsilon)=1-\sum_{k=1}^{n} \alpha_{k} \exp \left(-\varepsilon^{-1} \int_{0}^{\zeta_{k}} a(t) d t\right)$ is the function of $\varepsilon$ for $\varepsilon \in(0, \infty)$. Note that $\lim _{\varepsilon \rightarrow 0} \ell \omega(\varepsilon)=1$, but $\lim _{\varepsilon \rightarrow \infty} \ell \omega(\varepsilon)=1-\sum_{k=1}^{n} \alpha_{k}$. Since the function $\ell \omega(\varepsilon)$ is continuous on $(0, \infty)$, then it achieves zero at some value $\varepsilon=\varepsilon^{*} \in(0, \infty)$. Lemma 2.3 is proved.

Lemma 2.4. If all $\alpha_{k}$ have the same sign for $k=1,2, \cdots, n$, then NLIVP (1), (2)

(i) has not a critical parameter if $-\infty<\sum_{k=1}^{n} \alpha_{k} \leq 1$, i.e., the solution exists $\forall \varepsilon>0$;

(ii) has the unique critical parameter $\varepsilon^{*}$ if $\sum_{k=1}^{n} \alpha_{k}>1$, i.e., the corresponding solution exists for each $\varepsilon \neq \varepsilon^{*}$ and $\varepsilon^{*} \leq \varepsilon^{*} \leq \hat{\varepsilon}^{*}$ for

$$
\check{\varepsilon}^{*}=\int_{0}^{\zeta_{1}} a(t) d t / \ln \sum_{k=1}^{n} \alpha_{k}, \hat{\varepsilon}^{*}=\int_{0}^{\zeta_{n}} a(t) d t / \ln \sum_{k=1}^{n} \alpha_{k} .
$$


Proof. Denote ${ }^{3} \quad \alpha=\sum_{k=1}^{n} \alpha_{k}$. By virtue of Lemma 2.2 for the function $\ell \omega(\varepsilon)=1-\sum_{k=1}^{n} \alpha_{k} \exp \left(-\varepsilon^{-1} \int_{0}^{\zeta_{k}} a(t) d t\right)$ we shall check whether $\ell \omega(\varepsilon) \neq 0$ is true or not for the cases (i) and (ii).

(i). Since for $\omega(x)=\exp \left(-\varepsilon^{-1} \int_{0}^{x} a(t) d t\right)$ we have $\omega(0)=1, \omega(x)>0$ $\forall x \geq 0$ and $\omega(x)$ is a strictly decreasing continuous function, then

$$
\ell \omega(\varepsilon)> \begin{cases}1>0 & \text { for } \quad-\infty<\alpha \leq 0 \\ 1-\alpha \omega\left(\zeta_{1}\right)>0 & \text { for } \quad 0<\alpha \leq 1\end{cases}
$$

Therefore, NLIVP (1), (2) has not a critical parameter if $-\infty<\sum_{k=1}^{n} \alpha_{k} \leq 1$, i.e., the solution exists for each $\varepsilon>0$.

(ii). If $\alpha>1$, then by virtue of Lemma 2.3 the critical parameter exists. Since $[\ell \omega(\varepsilon)]^{\prime}=-\varepsilon^{-2} \sum_{k=1}^{n} \alpha_{k} \omega\left(\zeta_{k}\right) \int_{0}^{\zeta_{k}} a(t) d t$, then $[\ell \omega(\varepsilon)]^{\prime}<0$, so that $\ell \omega(\varepsilon)$ is a strictly decreasing on $(0, \infty)$. Therefore, $\ell \omega(\varepsilon)$ achieves zero at only one value $\varepsilon^{*} \in(0, \infty)$, i.e., the critical parameter is unique.

Put $\ell \omega(\varepsilon)=0$ to determine the location of $\varepsilon^{*}$. Using the mean value (MV) property [4, p. 1198] we obtain $\sum_{k=1}^{n} \alpha_{k} \omega\left(\zeta_{k}\right)=\alpha \omega\left(\zeta_{\varepsilon}\right)$ for each $\varepsilon>0$ and for an appropriate point $\zeta_{\varepsilon} \in\left[\zeta_{1}, \zeta_{n}\right]$. Since $\ell \omega(\varepsilon)=1-\alpha \omega\left(\zeta_{\varepsilon}\right)$, then from the equation $\ell \omega(\varepsilon)=0$ we get $\varepsilon^{*}=\int_{0}^{\zeta_{\varepsilon^{*}}} a(t) d t / \ln \alpha$ for some $\zeta_{\varepsilon^{*}} \in\left[\zeta_{1}, \zeta_{n}\right]$. Therefore,

$$
\int_{0}^{\zeta_{1}} a(x) d x / \ln \alpha \leq \varepsilon^{*} \leq \int_{0}^{\zeta_{n}} a(x) d x / \ln \alpha .
$$

Lemma 2.4 is proved.

Theorem 2.5. If all $\alpha_{k}$ have the same sign for $k=1,2, \cdots, n$, then for the classical solution of NLIVP (1), (2) the uniform on $\varepsilon$ and independent on $x a$ priori estimate holds

$$
|u(x)| \leq C\left(|\phi|+\max _{0 \leq t \leq X}|f(t)|\right), \quad 0 \leq x \leq X
$$

${ }^{3}$ Throughout the paper $\alpha$ denotes the sum $\sum_{k=1}^{n} \alpha_{k}$ by default. 
for each of the two cases:

(i) $-\infty<\alpha<1 \quad \forall \varepsilon>0 ; \quad \alpha=1, \quad 0<\varepsilon<\Upsilon, \forall \Upsilon>0$,

(ii) $1<\alpha, 0<\varepsilon \leq \check{\varepsilon}, \quad \check{\varepsilon}=\theta \zeta_{1} a / \ln \alpha \forall 0<\theta<1$.

Proof. The solution of NLIVP (1), (2) is represented by the sum $u(x)=v(x)$ $+w(x)$, so that $v(x)$ is a classical solution of the Cauchy problem

$$
\left\{\begin{array}{l}
L v(x)=f(x), \quad x>0, \\
v(0)=0
\end{array}\right.
$$

and $w(x)$ is a smooth solution of an appropriate NLIVP

$$
\left\{\begin{array}{l}
L w(x)=0, \quad x>0, \\
\ell w=\phi-\ell v .
\end{array}\right.
$$

For the classical solution of the Cauchy problem (4), the corresponding uniform on $\varepsilon$ a priori estimate holds [9, p. 13-14]:

$$
|v(x)| \leq \frac{1}{a} \max _{0 \leq t \leq X}|f(t)|, \quad 0 \leq x \leq X .
$$

Therefore,

$$
|\ell v| \leq \frac{|\alpha|}{a} \max _{0 \leq t \leq X}|f(t)| .
$$

Further, consider separately the cases (i) and (ii).

(i). By virtue of Lemma 2.4 the solution of NLIVP (1), (2) exists for all $\varepsilon>0$. Taking into account (6), to prove (3) it is sufficient to get a uniform on $\varepsilon$ a priori estimate for the solution of $\operatorname{NLIVP}(5)$. The solution of NLIVP (5) is represented by the formula

$$
w(x)=w(0) \omega(x),
$$

for $\omega(x)=\exp \left(-\varepsilon^{-1} \int_{0}^{x} a(t) d t\right)$, so that

$$
w(0)=(\ell \omega)^{-1}(\phi-\ell v)
$$

Since $\ell \omega(\varepsilon)=1-\sum_{k=1}^{n} \alpha_{k} \omega\left(\zeta_{k}\right)$, then

$$
\ell \omega(\varepsilon)> \begin{cases}1>0 & \text { for }-\infty<\alpha \leq 0, \\ 1-\alpha>0 & \text { for } 0<\alpha<1\end{cases}
$$


Therefore,

$$
|w(0)| \leq \begin{cases}|\phi|+|\ell v| & \text { for }-\infty<\alpha \leq 0 \\ (1-\alpha)^{-1}(|\phi|+|\ell v|) & \text { for } 0<\alpha<1\end{cases}
$$

Then taking into account (7) we get a uniform on $\varepsilon$ estimate

$$
|w(0)| \leq C_{1}\left(|\phi|+\max _{0 \leq t \leq X}|f(t)|\right)
$$

for $-\infty<\alpha<1$ and $C_{1}=$ const. Hence, using (8) and (9) we obtain a uniform on $\varepsilon$ a priori estimate for the solution of the problem (5)

$$
|w(x)| \leq C_{1}\left(|\phi|+\max _{0 \leq t \leq X}|f(t)|\right), 0 \leq x \leq X
$$

for $-\infty<\alpha<1$. Therefore, if $-\infty<\sum_{k=1}^{n} \alpha_{k}<1$, then a priori estimate successively follows from (6) and (10).

Put $\sum_{k=1}^{n} \alpha_{k}=1$. Then applying the MV property for the left-hand side of

we have $u(0)-u\left(\zeta_{\varepsilon}\right)=\phi$ for each $\varepsilon>0$ and for an appropriate $\zeta_{\varepsilon} \in\left[\zeta_{1}, \zeta_{n}\right]$. Therefore, $u(x)=v(x)+w(x)$, so that $v(x)$ is the solution of the Cauchy problem (4) and $w(x)$ is the solution of NLIVP (5) in terms of the two-point NLIVC (2) $\ell u \equiv u(0)-u\left(\zeta_{\varepsilon}\right)=\phi$. Since $u(x)$ and $v(x)$ exist, it is clear that $w(x)$ exists too. Because uniform on $\varepsilon$ a priori estimate (6) holds for $v(x)$, then

$$
|\ell v| \leq \frac{1}{a} \max _{0 \leq t \leq X}|f(t)|
$$

Since $\ell \omega=1-\omega\left(\zeta_{\varepsilon}\right)$, then taking into account the behavior of $\ell \omega(\varepsilon)$ and $\omega(x)$ (see Lemma 2.4) we obtain that $0<\check{\ell} \omega(\varepsilon)<\ell \omega(\varepsilon)$ for $\check{\ell} \omega(\varepsilon)=1-\omega\left(\zeta_{1}\right)$, where $\varepsilon \leq \Upsilon$ while an arbitrary real number $\Upsilon>0$. Therefore, $[\ell \omega(\varepsilon)]^{-1}$ $\leq[\check{\ell} \omega(\varepsilon)]^{-1}$. Since $\check{\ell} \omega(\varepsilon)$ is positive and a strictly decreasing on $(0, \infty)$, then from (9) it follows that

$$
|w(0)| \leq[\check{\ell} \omega(\Upsilon)]^{-1}(|\phi|+|\ell v|) .
$$

Taking into account (11) we get

$$
|w(0)| \leq C_{2}\left(|\phi|+\max _{0 \leq t \leq X}|f(t)|\right)
$$

for $C_{2}=[\check{\ell} \omega(\Upsilon)]^{-1} \max \{1,1 / a\}$. Hence by applying (8) we obtain a uniform on $\varepsilon$ a priori estimate (10) for the solution of NLIVP (5). Therefore, a uniform on $\varepsilon$ a priori estimate (3) holds if $\alpha=1$, which is the desired result. 
(ii). For $\alpha>1$ by virtue of Lemma 2.4 the NLIVP (1), (2) has some unique critical parameter $\varepsilon^{*}=\int_{0}^{\zeta_{\varepsilon}^{*}} a(x) d x / \ln \alpha$, so that $\int_{0}^{\zeta_{1}} a(x) d x / \ln \alpha \leq \varepsilon^{*}$. Therefore, the solution of NLIVP (1), (2) exists for all $\varepsilon \in(0, \check{\varepsilon}]$ since $\check{\varepsilon}<\varepsilon^{*}$ for $\check{\varepsilon}=\theta \zeta_{1} a / \ln \alpha, \quad 0<\theta<1$. This solution is represented by the sum $u(x)=v(x)+w(x)$, so that $v(x)$ and $w(x)$ are the corresponding solutions of the problems (4) and (5). Since (6) and (7) holds for $v(x)$, then it is sufficient to get a uniform on $\varepsilon$ a priori estimate for $w(x)$. Because $\ell \omega(\varepsilon)$ is a strictly decreasing and continuous function on $(0, \tilde{\varepsilon}], \lim _{\varepsilon \rightarrow 0} \ell \omega(\varepsilon)=1$ and $\ell \omega(\varepsilon)>0$ on $(0, \check{\varepsilon}]$, then taking into account (8) and (9) we get

$$
\mid w\left(0 \mid \leq[\ell \omega(\varepsilon)]^{-1}(|\phi|+|\ell v|) \leq[\ell \omega(\check{\varepsilon})]^{-1}(|\phi|+|\ell v|) .\right.
$$

Hence taking into account (7) we obtain uniform on $\varepsilon$ estimate

$$
|w(0)| \leq C_{3}\left(|\phi|+\max _{0 \leq t \leq X}|f(t)|\right)
$$

for $C_{3}=[\ell \omega(\check{\varepsilon})]^{-1} \max \{1, \alpha / a\}$. Using this estimate for $w(x)$ as for the classical solution of an appropriate Cauchy problem $L w(x)=0, \quad x>0, w(0)=\gamma$ we get a uniform on $\varepsilon$ and independent on $x$ a priori estimate

$$
|w(x)| \leq C_{4}\left(|\phi|+\max _{0 \leq t \leq X}|f(t)|\right), \quad 0 \leq x \leq X .
$$

Then a priori estimate (3) finally follows from (6) and (12). Theorem 2.5 is proved.

Corollary 2.6. If all $\alpha_{k}, k=1,2, \cdots, n$ have a positive sign and $\alpha>1$, then for the smooth solution of NLIVP (1), (2) uniform on $\varepsilon$ and independent on $x$ a priori estimation (3) holds for $\varepsilon \in(\hat{\varepsilon}, \infty)$, where $\hat{\varepsilon}=\Theta A \zeta_{n} / \ln \alpha, \Theta>1$ and $A=\max \{a(x) \forall x \in[0, X]\}$.

Proof. In extending the above proof we need only to observe that since $\varepsilon^{*}<\hat{\varepsilon}$, then $\ell \omega(\varepsilon)<\ell \omega(\hat{\varepsilon})<0$ for all $\varepsilon \in[\hat{\varepsilon}, \infty$ ) (see also the proof for case (ii) of Lemma 2.4) and therefore,

$$
\mid w\left(\left.0|\leq| \ell \omega(\varepsilon)\right|^{-1}(|\phi|+|\ell v|) \leq|\ell \omega(\hat{\varepsilon})|^{-1}(|\phi|+|\ell v|),\right.
$$

so that

$$
|w(0)| \leq C_{5}\left(|\phi|+\max _{0 \leq t \leq X}|f(t)|\right)
$$


for $C_{5}=|\ell \omega(\hat{\varepsilon})|^{-1} \max \{1, \alpha / a\}$. Using this inequality we get analogously to (12) a uniform on $\varepsilon$ and independent on $x$ a priori estimate. Corollary 2.6 is proved.

The following examples show some particularities concerning the proved statements.

Example 2.7. Exact critical parameter value. If $\alpha>1$ and $n=1$, then NLIVP (1), (2) with two-point value condition $u(0)-\alpha u(\zeta)=\phi$ has an exact critical parameter (see Lemma 2.4) $\varepsilon^{*}=\int_{0}^{\zeta} a(x) d x / \ln \alpha$. For example, the problem

$$
\varepsilon u^{\prime}(x)+u(x)=0, \quad x>0, \quad u(0)=\alpha u(\zeta)+1
$$

has solution $u(x)=[1-\alpha \exp (-\zeta / \varepsilon)]^{-1} \exp (-x / \varepsilon)$ if $1-\alpha \exp (-\zeta / \varepsilon) \neq 0$.

If $-\infty<\alpha<1$, then the solution is a uniform on $\varepsilon$ for all $\varepsilon>0$, so that $\lim _{\varepsilon \rightarrow \infty} u(x)=1 /(1-\alpha) \quad \forall x \geq 0$. However, if $\alpha=1$, then for arbitrary $x \geq 0 \quad \lim _{\varepsilon \rightarrow \infty} u(x)=+\infty$.

If $\alpha>1$, then the solution exists for $\varepsilon \neq \zeta / \ln \alpha$, i.e., $\varepsilon^{*}=\zeta / \ln \alpha$ is the exact critical parameter value. Particularly, if $\varepsilon \rightarrow \varepsilon^{*}-0$, then $u(x) \rightarrow$ $+\infty$, but if $\varepsilon \rightarrow \varepsilon^{*}+0$, then $u(x) \rightarrow-\infty$. In other words, the solution is collapsing, i.e., does not exists at $\varepsilon=\varepsilon^{*}$.

Example 2.8. Isolating interval for critical parameter. If $\alpha>1$ for all $\alpha_{k}>$ $0, k=1, \cdots, n$ and $n>1$, then NLIVP (1), (2) has some unique critical parameter (Lemma 2.4), but it is not evaluated exactly. Actually, in order to avoid such critical parameter we have to isolate it. In view of Theorem 2.5 and Corollary 2.6 we choose $\check{\varepsilon}=\theta \zeta_{1} a / \ln \alpha$ for $0<\theta<1$, $\hat{\varepsilon}=\Theta A / \ln \alpha$ for $\Theta>1$ and $A=\max \{a(x) \quad \forall x \in[0, X]\}$ to determine the isolating interval $(\check{\varepsilon}, \hat{\varepsilon})$.

For example, if $\phi+\alpha_{1}+\alpha_{2}-1 \neq 0$, then the problem

$$
\varepsilon u^{\prime}(x)+u(x)=1, \quad x>0, \quad u(0)-\alpha_{1} u\left(\zeta_{1}\right)-\alpha_{2} u\left(\zeta_{2}\right)=\phi
$$

has different from $u(x) \equiv 1$ unique solution

$$
u(x)=\left[1-\alpha_{1} \exp \left(-\zeta_{1} / \varepsilon\right)-\alpha_{2} \exp \left(-\zeta_{2} / \varepsilon\right)\right]^{-1}\left(\phi+\alpha_{1}+\alpha_{2}-1\right) \exp (-x / \varepsilon)+1
$$

if $1-\alpha_{1} \exp \left(-\zeta_{1} / \varepsilon\right)-\alpha_{2} \exp \left(-\zeta_{2} / \varepsilon\right) \neq 0$. Put $\alpha=\alpha_{1}+\alpha_{2}>1$ for $\alpha_{1}>0$ and $\alpha_{2}>0$, then formally

$$
u(x)=\left[1-\alpha \exp \left(-\zeta_{\varepsilon} / \varepsilon\right)\right]^{-1}(\phi+\alpha-1) \exp (-x / \varepsilon)+1
$$


for an appropriate $\zeta_{\varepsilon} \in\left[\zeta_{1}, \zeta_{2}\right]$. Hence for this critical parameter we have implicit expression $\varepsilon^{*}=\zeta_{\varepsilon^{*}} / \ln \alpha$ which does not allow to find its value exactly. However, we can bound it by an isolating interval if select $\check{\varepsilon}=\theta \zeta_{1} / \ln \alpha, \quad 0<\theta<1$ and $\hat{\varepsilon}=\Theta \zeta_{2} / \ln \alpha, \Theta>1$. Outside of the isolating interval $(\check{\varepsilon}, \hat{\varepsilon})$ the solution exists and is a uniform on $\varepsilon$.

\section{Multipoint problem with NLIVC having different signs factors}

We suppose that NLIVP (1), (2) has different signs for the factors $\alpha_{k} \quad(k=$ $1, \cdots, n)$ and reformulate the problem for convenience without losing in generality.

Consider the following NLIVP

$$
\begin{aligned}
L u & \equiv \varepsilon u^{\prime}(x)+a(x) u(x)=f(x), \quad x>0, \\
\ell u & \equiv u(0)-\sum_{k=1}^{n} \alpha_{k} u\left(\zeta_{k}\right)+\sum_{l=1}^{m} \beta_{l} u\left(\eta_{l}\right)=\phi
\end{aligned}
$$

for smooth functions $f(x)$ and $a(x), a(x) \geq a>0$ for $x \geq 0, \varepsilon>0, \alpha_{k}>$ $0, k=1, \cdots, n$ and $\beta_{l}>0, l=1, \cdots, m, \quad 0<\zeta_{1}<\zeta_{2}<\cdots<\zeta_{n}<X$, $0<\eta_{1}<\eta_{2}<\cdots<\eta_{m}<X, \quad X \in R, \quad \phi \in R$. In view of (14), we denote the function

$$
\ell \omega(\varepsilon)=1-\sum_{k=1}^{n} \alpha_{k} \exp \left(-\varepsilon^{-1} \int_{0}^{\zeta_{k}} a(t) d t\right)+\sum_{l=1}^{m} \beta_{l} \exp \left(-\varepsilon^{-1} \int_{0}^{\eta_{l}} a(t) d t\right) .
$$

Following is the reformulation of Lemma 2.3.

Lemma 3.1. If $\sum_{k=1}^{n} \alpha_{k}-\sum_{l=1}^{m} \beta_{l}>1$, then $\operatorname{NLIVP}(13)$, (14) has some critical parameter $\varepsilon^{*}>0$.

Proof. Since $\lim _{\varepsilon \rightarrow 0} \ell \omega(\varepsilon)=1$ but $\lim _{\varepsilon \rightarrow \infty} \ell \omega(\varepsilon)=1-\sum_{k=1}^{n} \alpha_{k}+\sum_{l=1}^{m} \beta_{l}$, then the continuous on $(0, \infty)$ function $\ell \omega(\varepsilon)$ achieves zero at some $\varepsilon^{*} \in(0, \infty)$ if $\sum_{k=1}^{n} \alpha_{k}-\sum_{l=1}^{m} \beta_{l}>1$. Lemma 3.1 is proved.

Lemma 3.2. The classical solution of NLIVP (13), (14) exists for each of the following cases under corresponding requirements: ${ }^{4}$

${ }^{4}$ Throughout the paper, the symbol $\alpha$ denotes the sum $\sum_{k=1}^{n} \alpha_{k}$ and the symbol $\beta$ denotes $\sum_{l=1}^{m} \beta_{l}$ by default. 
(i) $\alpha \leq 1, \quad \forall \varepsilon>0$;

(ii) $-\infty<\alpha-\beta \leq 1, \quad \eta_{m}<\zeta_{1} \quad \forall \varepsilon>0$;

(j) $\quad 1<\alpha-\beta, \quad \eta_{m}<\zeta_{1}, \quad \varepsilon \in(0, \check{\varepsilon}] \cup[\hat{\varepsilon}, \infty)$,

$\check{\varepsilon}=\theta\left(\zeta_{1}-\eta_{m}\right) a / \ln (\alpha /(1+\beta)) \quad \forall 0<\theta<1$,

$\hat{\varepsilon}=\Theta \zeta_{n} A / \ln (\alpha /(1+\beta)) \forall \Theta>1 ;$

(jj) $1<\alpha-\beta, \quad \zeta_{n}<\eta_{1}, \quad \varepsilon \in(0, \check{\varepsilon}] \cup[\hat{\varepsilon}, \infty)$,

$\check{\varepsilon}=\theta \zeta_{1} a / \ln \alpha \quad \forall 0<\theta<1$,

$\hat{\varepsilon}=\Theta \eta_{m} A / \ln (\alpha-\beta) \forall \Theta>1 ;$

(jjj) $1<\alpha, \quad \varepsilon \in(0, \check{\varepsilon}], \quad \check{\varepsilon}=\theta \zeta_{1} a / \ln \alpha \quad \forall 0<\theta<1$.

Proof. In view of Lemma 2.2 we shall check whether or not $\ell \omega(\varepsilon) \neq 0$ to determine the existence of a solution of NLIVP (13),(14), where

$$
\ell \omega(\varepsilon)=1-\sum_{k=1}^{n} \alpha_{k} \omega\left(\zeta_{k}\right)+\sum_{l=1}^{m} \beta_{l} \omega\left(\eta_{l}\right), \quad \omega(x)=\exp \left(-\varepsilon^{-1} \int_{0}^{x} a(t) d t\right) .
$$

(i). Taking into account the behavior of $\omega(x)$ we have the following inequalities $\forall \varepsilon>0$

$$
\ell \omega(\varepsilon)> \begin{cases}1-\alpha>1>0 & \text { for } \quad 0<\alpha<1 \\ 1-\omega\left(\zeta_{1}\right)>0 & \text { for } \quad \alpha=1\end{cases}
$$

regardless of the location of the points $\zeta_{k}, k=1, \cdots, n$ and $\eta_{l}, l=1, \cdots, m$. It means that the solution exists for $\forall \varepsilon>0$ when $\alpha \leq 1$.

(ii). If $\eta_{m}<\zeta_{1}$, then $\forall \varepsilon>0$ we have

$$
\ell \omega(\varepsilon)> \begin{cases}1-(\alpha-\beta)>1>0 & \text { for }-\infty<\alpha-\beta \leq 0, \\ 1-(\alpha-\beta)>0 & \text { for } 0<\alpha-\beta<1, \\ 1-\omega\left(\eta_{m}\right)>0 & \text { for } \alpha-\beta=1 .\end{cases}
$$

Therefore, the solution exists for case (ii).

(j). Since $\alpha-\beta>1$, some critical parameter exists in view of the Lemma 3.1. By virtue of the MV property

$$
\ell \omega(\varepsilon)=1-\alpha \omega\left(\zeta_{\varepsilon}\right)+\beta \omega\left(\eta_{\varepsilon}\right)=(1+\beta) \omega\left(\xi_{\varepsilon}\right)-\alpha \omega\left(\zeta_{\varepsilon}\right)
$$

for each $\varepsilon>0$ and an appropriate $\xi_{\varepsilon} \in\left[0, \eta_{\varepsilon}\right], \eta_{\varepsilon} \in\left[\eta_{1}, \eta_{m}\right], \zeta_{\varepsilon} \in\left[\zeta_{1}, \zeta_{n}\right]$. Since $\eta_{m}<\zeta_{1}$, from the equation $\ell \omega(\varepsilon)=0$ we get a formal expression for the critical parameter $\varepsilon^{*}=[\ln \alpha-\ln (1+\beta)]^{-1} \int_{\varepsilon^{*}}^{\zeta^{*}} a(x) d x$. Then $\varepsilon^{*} \in(\check{\varepsilon}, \hat{\varepsilon})$ for $\check{\varepsilon}=\theta\left(\zeta_{1}-\eta_{m}\right) a / \ln (\alpha /(1+\beta)) \quad \forall 0<\theta<1$ and $\hat{\varepsilon}=\Theta \zeta_{n} A / \ln (\alpha /(1+\beta))$ $\forall \Theta>1$ with $A=\max \{a(x) \forall x \in[0, X]\}$. Therefore, the solution always exists outside of $(\check{\varepsilon}, \hat{\varepsilon})$, i.e., the theorem statement for case (j) is proved. 
(jj). Since $\alpha-\beta>1$, some critical parameter exists by virtue of the Lemma 3.1. For an arbitrary $\varepsilon>0 \quad \ell \omega(\varepsilon)>1-\alpha \omega\left(\zeta_{\varepsilon}\right)$ for $\zeta_{\varepsilon} \in\left[\zeta_{1}, \zeta_{n}\right]$. If for some $\varepsilon$ the inequality $1-\alpha \omega\left(\zeta_{\varepsilon}\right)>0$ holds, then the inequality $\ell \omega(\varepsilon)>0$ also holds. Similarly, if for some $\varepsilon 1-\alpha \omega\left(\zeta_{1}\right)>0$, then $1-\alpha \omega\left(\zeta_{\varepsilon}\right)>0$ since $1-\alpha \omega\left(\zeta_{\varepsilon}\right)>1-\alpha \omega\left(\zeta_{1}\right)$. Actually, $1-\alpha \omega\left(\zeta_{1}\right)>0$ for all $\varepsilon \in(0, \tilde{\varepsilon}]$ if $\check{\varepsilon}=\theta \zeta_{1} a / \ln \alpha \forall 0<\theta<1$ and $\alpha>1+\beta$. Therefore, $\ell \omega(\varepsilon)>0$ for $\varepsilon \in(0, \check{\varepsilon}]$, it means that the solution exists for $\varepsilon \in(0, \check{\varepsilon}]$.

On the other hand, $\ell \omega(\varepsilon)<1-(\alpha-\beta) \omega\left(\eta_{\varepsilon}\right)<1-(\alpha-\beta) \omega\left(\eta_{m}\right)$ for $1<\alpha-\beta$. If $1-(\alpha-\beta) \omega\left(\eta_{m}\right)<0$, then $\ell \omega(\varepsilon)<0$ too. Since 1 $-(\alpha-\beta) \omega\left(\eta_{m}\right)<0$ for $\varepsilon>\int_{0}^{\eta_{m}} a(x) / \ln (\alpha-\beta) d x$, then $\ell \omega(\varepsilon)<0$ for all $\varepsilon \in[\hat{\varepsilon}, \infty)$ if $\hat{\varepsilon}=\Theta \eta_{m} A / \ln (\alpha-\beta) \forall \Theta>1$. It means that the solution exists for $\varepsilon \in[\hat{\varepsilon}, \infty)$.

In summary, the solution exists for all $\varepsilon$ laying outside of the interval $(\check{\varepsilon}, \hat{\varepsilon})$. The theorem statement for case $(\mathrm{jj})$ is proved.

(jjj) Here is $\ell \omega(\varepsilon)>1-\alpha \omega\left(\zeta_{1}\right)$. Since $1-\alpha \omega\left(\zeta_{1}\right)>0$ for $\varepsilon \in(0, \check{\varepsilon}]$ where $\check{\varepsilon}=\theta \zeta_{1} a / \ln \sum_{k=1}^{n} \alpha_{k} \quad \forall 0<\theta<1$, then $\ell \omega(\varepsilon)>0$ for all $\varepsilon \in(0, \check{\varepsilon}]$. Therefore, the solution exists for case (jjj). Lemma 3.2 is proved.

Theorem 3.3. For each case of Lemma 3.2, but with additional restriction in the case

(ii): $0<\varepsilon \leq \Upsilon, \forall \Upsilon>0$ when $\alpha-\beta=1$,

for the classical solution of NLIVP (13), (14) a uniform on $\varepsilon$ and independent on $x$ a priori estimation (3) holds.

Proof. Put $u(x)=v(x)+w(x)$, so that $v(x)$ is the classical solution of the Cauchy problem

$$
L v(x)=f(x), x>0, v(0)=0
$$

and $w(x)$ is a smooth solution of NLIVP

$$
L w(x)=0, x>0, \quad \ell w=\phi-\ell v .
$$

Since for the classical solution of the Cauchy problem a uniform on $\varepsilon$ a priori estimation (6) holds, then

$$
|\ell v| \leq \frac{\alpha+\beta}{a} \max _{0 \leq t \leq X}|f(t)|
$$


On the other hand, for $w(x)$ we have

$$
w(x)=w(0) \exp \left(-\frac{1}{\varepsilon} \int_{0}^{x} a(t) d t\right), \quad w(0)=(\ell \omega)^{-1}(\phi-\ell v),
$$

where $\omega(x)=w(x) / w(0)$ for all $x \geq 0$.

(i). Regardless the location of the points $\zeta_{k}, k=1, \cdots, n$ and $\eta_{l}, l=$ $1, \cdots, m, \quad \forall \varepsilon>0$ the following inequalities hold:

$$
|w(0)| \leq(\ell \omega)^{-1}(|\phi|+|\ell v|) \leq(1-\alpha)^{-1}(|\phi|+|\ell v|) \text { if } 0<\alpha<1 .
$$

Hence taking into account (15) we have

$$
|w(0)| \leq C_{1}\left(|\phi|+\max _{0 \leq t \leq X}|f(t)|\right), \quad 0 \leq x \leq X
$$

Therefore, for $w(x)$ as for the classical solution of an appropriate Cauchy problem

$$
L w(x)=0, \quad x>0, w(0)=\gamma,
$$

a uniform on $\varepsilon$ and independent on $x$ a priori estimate holds

$$
|w(x)| \leq C_{2}\left(|\phi|+\max _{0 \leq t \leq X}|f(t)|\right), \quad 0 \leq x \leq X
$$

Then a priori estimate (3) successively follows from (6) and (17) for the subcase when $0<\alpha<1$.

If $\alpha=1$, then positive and continuous on $(0, \infty)$ function $\ell \omega(\varepsilon)$ attains the minimum at some value of $\varepsilon \in(0, \infty)$ because $\lim _{\varepsilon \rightarrow 0} \ell \omega(\varepsilon)=1$ and $\lim _{\varepsilon \rightarrow \infty} \ell \omega(\varepsilon)=\beta$. Therefore,

$$
|w(0)| \leq[\min \ell \omega(\varepsilon)]^{-1}(|\phi|+|\ell v|),
$$

then (16) and (17) actually follow. A priori estimate (3) is proved for (i).

(ii). For $\eta_{m}<\zeta_{1}$ and $\forall \varepsilon>0$ we have the sequence of inequalities

$$
\begin{gathered}
|w(0)| \leq(\ell w)^{-1}(|\phi|+|\ell v|) \leq\left[1-(\alpha-\beta) \omega\left(\eta_{m}\right)\right]^{-1}(|\phi|+|\ell v|) \\
\quad \leq \begin{cases}(|\phi|+|\ell v|), & \text { if }-\infty<\alpha-\beta \leq 0, \\
(1-\alpha+\beta)^{-1}(|\phi|+|\ell v|), & \text { if } 0<\alpha-\beta<1 .\end{cases}
\end{gathered}
$$


Hence, by taking into account (6) and (15), we get a priori estimation (3) if $-\infty<\alpha-\beta<1$.

If $\alpha-\beta=1$, then since the critical parameter does not exist (Lemma 3.2) the continuous function $\ell \omega(\varepsilon)$ is positive on $(0, \infty)$. Then the function $\ell \omega(\varepsilon)$ attains the minimum at some value $\varepsilon \in(0, \Upsilon]$ for arbitrary $\Upsilon>0$ because $\lim _{\varepsilon \rightarrow 0} \ell \omega(\varepsilon)=1$ and $\lim _{\varepsilon \rightarrow \infty} \ell \omega(\varepsilon)=0$. It means that we sequentially obtain (18), (16) and (17). Therefore, a priori estimate (3) is proved for (ii).

(j)-(jjj). Because $\lim _{\varepsilon \rightarrow 0} \ell \omega(\varepsilon)=1$ but $\lim _{\varepsilon \rightarrow \infty} \ell \omega(\varepsilon)=1-\alpha+\beta$, then the continuous on $(0, \infty)$ function $\ell \omega(\varepsilon)$ attains zero at some critical parameter $\varepsilon^{*} \in(\check{\varepsilon}, \hat{\varepsilon})$ (Lemma 3.2) for cases (j) and (jj). In view of isolating intervals we have the corresponding sequence of inequalities

$$
\begin{gathered}
|w(0)| \leq|\ell \omega(\varepsilon)|^{-1}(|\phi|+|\ell v|) \\
\leq \begin{cases}\min _{0<\varepsilon \leq \check{\varepsilon}}[\ell \omega(\varepsilon)]^{-1}(|\phi|+|\ell v|) & \text { for } \varepsilon \in(0, \check{\varepsilon}], \\
{\left[\min _{\hat{\varepsilon} \leq \varepsilon<\infty}|\ell \omega(\varepsilon)|\right]^{-1}(|\phi|+|\ell v|)} & \text { for } \varepsilon \in[\hat{\varepsilon}, \infty)\end{cases}
\end{gathered}
$$

for each of the cases (j) and (jj). Applying these inequalities we successively obtain (18), (16) and (17).

Eventually, if some critical parameter exists for the case (jjj), then $\varepsilon^{*} \in$ $(\check{\varepsilon}, \infty)$ (see Lemma 3.2). Therefore,

$$
|w(0)| \leq|\ell \omega(\varepsilon)|^{-1}(|\phi|+|\ell v|) \leq \min _{0<\varepsilon \leq \check{\varepsilon}}[\ell \omega(\varepsilon)]^{-1}(|\phi|+|\ell v|)
$$

for $\varepsilon \in(0, \check{\varepsilon}]$. Since outside of the isolating interval $(\check{\varepsilon}, \infty)$ the function $\ell \omega(\varepsilon)$ is bounded from below, then from the above inequalities we receive (18), (16), (17) for the case (jjj) too.

In summary, it means that a priori estimate (3) is established for all cases of the original theorem. Theorem 3.3 is proved.

Example 3.4. Essentiality of the restriction on $\varepsilon$ if $\alpha-\beta=1$ for (ii). The function $u(x)=[1-\alpha \exp (-\zeta / \varepsilon)+\beta \exp (-\eta / \varepsilon)]^{-1} \exp (-x / \varepsilon)$ represents the solution of the problem

$$
\varepsilon u^{\prime}(x)+u(x)=0, \quad x>0, \quad u(0)-\alpha u(\zeta)+\beta u(\eta)=1
$$

for $\forall \varepsilon>0$ if $-\infty<\alpha-\beta \leq 1$ for $\alpha>0$ and $\beta>0$. Actually,

$$
\lim _{\varepsilon \rightarrow \infty} u(x)= \begin{cases}(1-\alpha+\beta)^{-1} & \text { if }-\infty<\alpha-\beta<1, \\ +\infty & \text { if } \alpha-\beta=1 .\end{cases}
$$


We observe, that the additional restriction on $\varepsilon$ for the case (ii) when $\alpha-\beta=1$ is essential. Put $\beta=0$, then the example shows the essentiality of the additional bound on $\varepsilon$ for the case (i) of Theorem 2.5 when $\alpha=1$.

Example 3.5. Essentiality of the nonlocal points location for (ii). First, put $\zeta<\eta$, but nevertheless let $\alpha-\beta<1$ for the problem

$$
\varepsilon u^{\prime}(x)+u(x)=0, \quad x>0, \quad u(0)-\alpha u(\zeta)+\beta u(\eta)=1
$$

with $\alpha>0, \beta>0$. For example, let $\varepsilon=1, \alpha=e, \zeta=1 / 2$ and $\beta=1000$. Choose $\eta=\ln (\beta /(\alpha \exp (-\zeta)-1))=7.340504 \ldots$, then $\varepsilon^{*}=1$ is the critical parameter. We observe that nevertheless $\alpha-\beta<1$, the critical parameter exists if the layout of $\zeta$ and $\eta$ contradicts with Theorem 3.3 requirements.

Second, put $\zeta<\eta$ but yet $\alpha-\beta=1$ for above problem. Put $\varepsilon=1$, $\alpha=e, \zeta=1 / 2, \beta=e-1$. Then $\varepsilon^{*}=1$ is the critical parameter if $\eta=$ $\ln (\beta / \alpha \exp (-\zeta)-1)=0.97407 \ldots$. It is clear that in Theorem 3.3 the restriction on the layout of $\zeta$ and $\eta$ is essential for the case if $\alpha-\beta=1$ too.

Example 3.6. Isolating interval. For $\alpha>0, \beta>0, \eta<\zeta$, but $\alpha-\beta>1$ the problem

$$
\varepsilon u^{\prime}(x)+u(x)=1, \quad x>0, \quad u(0)-\alpha u(\zeta)+\beta u(\eta)=0
$$

has the solution

$$
u(x)=(\alpha-\beta-1)[1-\alpha \exp (-\zeta / \varepsilon)+\beta \exp (-\eta / \varepsilon)]^{-1} \exp (-x / \varepsilon)+1
$$

if $\ell \omega(\varepsilon)=1-\alpha \exp (-\zeta / \varepsilon)+\beta \exp (-\eta / \varepsilon) \neq 0$. However, yet it is possible that $\ell \omega(\varepsilon)=0$. Then the equality $(1+\beta) \omega\left(\xi_{\varepsilon}\right)=\alpha \omega(\zeta)$ holds for $\xi_{\varepsilon} \in(0, \eta)$. Hence the formula $\varepsilon^{*}=\left(\zeta-\xi_{\varepsilon^{*}}\right) /(\ln \alpha-\ln (1+\beta))$ formally determines some critical parameter because $\ell \omega\left(\varepsilon^{*}\right)=0$. With regard to the statement $(j)$ of Lemma 3.2, we get

$$
\varepsilon^{*} \in[(\zeta-\eta) /(\ln \alpha-\ln (1+\beta)), \quad \zeta /(\ln \alpha-\ln (1+\beta))] \quad \text { if } \alpha-\beta>1 .
$$

To avoid the collapsing of the solution we choose $\check{\varepsilon}=\theta(\zeta-\eta) /[\ln \alpha-\ln (1+\beta)]$ and $\hat{\varepsilon}=\Theta \zeta /[\ln \alpha-\ln (1+\beta)]$ for $0<\theta<1, \quad \Theta>1$, so that the solution always exists outside of the isolating interval $(\check{\varepsilon}, \hat{\varepsilon})$.

Corollary 3.7. If $m=n, \eta_{1} \leq \zeta_{1}<\eta_{2} \leq \zeta_{2}<\cdots<\eta_{n} \leq \zeta_{n}$ and $\sum_{k=1}^{n}\left(\alpha_{k}-\beta_{k}+\left|\alpha_{k}-\beta_{k}\right|\right) / 2 \leq 1$, then the classical solution of NLIVP (13), (14) exists for all $\varepsilon>0$ and a uniform on $\varepsilon$ a priori estimation (3) holds for the cases: 
(i) $\forall \varepsilon>0$ if $\sum_{k=1}^{n}\left(\alpha_{k}-\beta_{k}+\left|\alpha_{k}-\beta_{k}\right|\right) / 2<1$,

(j) $\forall \varepsilon>0$ if $\sum_{k=1}^{n}\left(\alpha_{k}-\beta_{k}+\left|\alpha_{k}-\beta_{k}\right|\right) / 2=1$ but $\sum_{k=1}^{n}\left(\alpha_{k}-\beta_{k}-\mid \alpha_{k}\right.$ $\left.-\beta_{k} \mid\right) / 2 \neq 0$,

(jj) for $0<\varepsilon \leq \Upsilon, \quad \forall \Upsilon>0$ if $\sum_{k=1}^{n}\left(\alpha_{k}-\beta_{k}+\left|\alpha_{k}-\beta_{k}\right|\right) / 2=1$ but $\sum_{k=1}^{n}\left(\alpha_{k}-\beta_{k}-\left|\alpha_{k}-\beta_{k}\right|\right) / 2=0$.

Proof. The proof is similar to the proof of Lemma 3.2 and Theorem 3.3. Corollary 3.4 is proved.

Theorem 3.8. If $n=m=1$ and $\alpha-\beta>1$, then NLIVP (13),(14) has not more than only one critical parameter.

Proof. If $\alpha-\beta>1$, then by virtue of Lemma 3.1 some critical parameter exists.

Suppose that more than one critical parameters exist. Since $\lim _{\varepsilon \rightarrow 0} \ell \omega(\varepsilon)=1$ and $\lim _{\varepsilon \rightarrow \infty} \ell \omega(\varepsilon)=1-\alpha+\beta$, then the continuous on $(0, \infty)$ function $\ell \omega(\varepsilon)$ has equal to zero derivative $[\ell \omega(\varepsilon)]^{\prime}=0$ at least for two different values of $\varepsilon$. Since

$$
[\ell \omega(\varepsilon)]^{\prime}=\varepsilon^{2}\left[\beta \omega(\eta) \int_{0}^{\eta} a(t) d t-\alpha \omega(\zeta) \int_{0}^{\zeta} a(t) d t\right],
$$

then $\beta \omega(\eta) \int_{0}^{\eta} a(t) d t=\alpha \omega(\zeta) \int_{0}^{\zeta} a(t) d t$ at least for two different values of $\varepsilon$.

Let $\eta<\zeta$. Then $\omega(\zeta) / \omega(\eta)=\Xi$ for $\Xi=\beta \int_{0}^{\eta} a(t) d t /\left[\alpha \int_{0}^{\zeta} a(t) d t\right]$ since $\eta<\zeta$ and $\alpha>\beta$. Therefore, $\exp \left(-\varepsilon^{-1} \int_{\eta}^{\zeta} a(t) d t\right)=\Xi$. Hence we get the formula $\varepsilon=-\int_{\eta}^{\zeta} a(t) d t / \ln \Xi$ for $\varepsilon$ satisfying $[\ell \omega(\varepsilon)]^{\prime}=0$. However, this formula determines only a single value $\varepsilon$ which can satisfy $[\ell \omega(\varepsilon)]^{\prime}=0$. We thereby reach a contradiction.

Put $\zeta<\eta$. Then formally $\omega(\eta) / \omega(\zeta)=\Xi$ for $\Xi=\alpha \int_{0}^{\zeta} a(t) d t /\left[\beta \int_{0}^{\eta} a(t) d t\right]$. If $\Xi \geq 1$, then $\varepsilon$ cannot satisfy $[\ell \omega(\varepsilon)]^{\prime}=0$ and this state contradicts to the assumption that $[\ell \omega(\varepsilon)]^{\prime}=0$ at least for two different values of $\varepsilon$. If $\Xi<1$, 
then we get the formula $\varepsilon=-\int_{\zeta}^{\eta} a(t) d t / \ln \Xi$ for $\varepsilon$ satisfying $[\ell \omega(\varepsilon)]^{\prime}=0$. Since this formula determines only a single value $\varepsilon$ which satisfies $[\ell \omega(\varepsilon)]^{\prime}=0$, then thereby we reach a contradiction once again. Theorem 3.5 is proved.

\section{Integral kind of NLIVP}

Consider NLIVP with the integral condition

$L u(x) \equiv \varepsilon u^{\prime}(x)+a(x) u(x)=f(x), \quad x>0, \quad \ell u \equiv u(0)-\int_{\zeta_{1}}^{\zeta_{2}} \alpha(x) u(x) d x=\phi$ for $0<\zeta_{1}<\zeta_{2}<X$ and $\alpha(x) \in L_{2}\left(\zeta_{1}, \zeta_{2}\right)$. If $\alpha(x)$ does not change sign almost everywhere on $\left[\zeta_{1}, \zeta_{2}\right]$, then Lemma 2.4 and Theorem 2.5 are aplicable to the research this kind of NLIVP.

Indeed, by virtue of the mean value theorem the nonlocal integral condition is reducible to the two-point NLIVC: $u(0)-\alpha u(\zeta)=\phi$ for $\alpha=\int_{\zeta_{1}}^{\zeta_{2}} \alpha(x) d x$ and for an appropriate $\zeta \in\left[\zeta_{1}, \zeta_{2}\right]$. Further, we consider the solution of NLIVP with integral kind of NLIVC as the solution of the corresponding twopoint NLIVP. By virtue of Theorem 2.5 we use the theorem statement (i) if

$-\infty<\int_{\zeta_{1}}^{\zeta_{2}} \alpha(x) d x \leq 1$ or the alternative statement (ii) if $1<\int_{\zeta_{1}}^{\zeta_{2}} \alpha(x) d x$. A possible critical parameter is not exact if $1<\int_{\zeta_{1}}^{\zeta_{2}} \alpha(x) d x$, however an appropriate isolating interval is easily determinable.

Consider NLIVP with a complicated integral condition:

$L u(x)=f(x), \quad x>0, \quad \ell u \equiv u(0)-\int_{\zeta_{1}}^{\zeta_{2}} \alpha(x) u(x) d x+\int_{\eta_{1}}^{\eta_{2}} \beta(x) u(x) d x=\phi$,

$0<\zeta_{1}<\zeta_{2}<X, 0<\eta_{1}<\eta_{2}<X, \alpha(x) \in L_{2}\left(\zeta_{1}, \zeta_{2}\right), \beta(x) \in L_{2}\left(\eta_{1}, \eta_{2}\right)$, $\int_{\zeta_{1}}^{\zeta_{2}} \alpha(x) d x>0, \int_{\eta_{1}}^{\eta_{2}} \beta(x) d x>0$ for $\alpha(x) \geq 0$ almost everywhere on $\left[\zeta_{1}, \zeta_{2}\right]$ and $\beta(x) \geq 0$ almost everywhere on $\left[\eta_{1}, \eta_{2}\right]$. By virtue of the mean value theorem this nonlocal integral condition is reducible to the three-point NLIVC: $u(0)-\alpha u(\zeta)+\beta u(\eta)=\phi$ for $\alpha=\int_{\zeta_{1}}^{\zeta_{2}} \alpha(x) d x, \zeta \in\left[\zeta_{1}, \zeta_{2}\right]$ and $\beta=\int_{\eta_{1}}^{\eta_{2}} \beta(x) d x$, 
$\eta \in\left[\eta_{1}, \eta_{2}\right]$. In view of Lemma 3.2 and Theorem 3.3 we assign the following cases:

(i) $0<\int_{\zeta_{1}}^{\zeta_{2}} \alpha(x) d x \leq 1$

(ii) $-\infty<\int_{\zeta_{1}}^{\zeta_{2}} \alpha(x) d x-\int_{\eta_{1}}^{\eta_{2}} \beta(x) d x \leq 1, \quad \eta_{2}<\zeta_{1}$.

Further we apply statements (i) and (ii) of Lemma 3.2 and Theorem 3.3 appropriately. Additionally, we can assign other cases corresponding to the cases $(j)-(j j j)$ of Lemma 3.2 and Theorem 3.3 and apply appropriate statements to research such problems with integral kind of NLIVC.

\section{Asymptotic expansion of a solution of NLIVP}

Here we prove that for a smooth solution of NLIVP an asymptotic expansion in terms of a small parameter $\varepsilon$ holds.

Theorem 5.1. Let $a(x)$ and $f(x)$ be sufficiently smooth functions. Let $u(x)$ be a smooth solution of NLIVP which is considered in view of Theorem 2.5, Corollary 2.6, Theorem 3.3 or Corollary 3.7. Then for a sufficiently small parameter $\varepsilon>0$ the solution $u(x)$ has an asymptotic expansion

$$
u(x)=\sum_{k=0}^{M} \varepsilon^{k}\left(u_{k}(x)+v_{k}(\tau)\right)+R_{\varepsilon, M}, \quad \tau=x / \varepsilon
$$

with $\left|R_{\varepsilon, M}\right| \leq C \varepsilon^{M+1}$ and an independent on $\varepsilon$ constant $C$, so that

$$
\begin{gathered}
a(x) u_{0}(x)=f(x) \quad \forall x \geq 0 ; \quad a(x) u_{i}(x)=-u_{i-1}^{\prime}(x), i=\overline{1, M} \quad \forall x \geq 0 ; \\
\quad \frac{d}{d \tau} v_{0}(\tau)+a(0) v_{0}(\tau)=0, \quad \tau>0, \quad \ell v_{0}=\phi-\ell u_{0} ; \\
\frac{d}{d \tau} v_{i}(\tau)+a(0) v_{i}(\tau)=s_{i}(\tau), \quad \tau>0, \quad \ell v_{i}=-\ell u_{i}, \\
s_{i}(\tau)=\sum_{k=0}^{i-1} \frac{\tau^{i-k}}{(i-k) !} a^{(i-k)}(0) v_{k}(\tau), \quad \tau>0, \quad i=\overline{1, M} .
\end{gathered}
$$

Proof. Similar to $[9$, p. 15] we have to write an appropriate differential problem for the remainder $R_{\varepsilon, M}$ by using the desired expansion but with the difference that instead of initial data for $v_{i}(0), i=0,1, \cdots, M$ we have nonlocal conditions correspondingly. Actually, the remainder $R_{\varepsilon, M}$ represents the solution of NLIVP

$$
L R_{\varepsilon, M}(x)=\varepsilon^{M+1} g(x), \quad x>0, \quad \ell R_{\varepsilon, M}=0 .
$$


The function $g(x)$ is definable by virtue of the conditions of the theorem as a linear combination of continuous functions and functions

$$
v_{i}(\tau)=P_{2 i}(\tau) \exp (-a(0) \tau)
$$

These $P_{2 i}$ are an appropriate polynomials of degree $2 i$. The solution of (19) is represented by the sum $R_{\varepsilon, M}(x)=v(x)+w(x)$ for $v(x)$ satisfying the Cauchy problem

$$
L v(x) \equiv \varepsilon v^{\prime}(x)+a(x) v(x)=\varepsilon^{M+1} g(x), \quad x>0, \quad v(0)=0
$$

and $w(x)$ satisfying the NLIVP

$$
L w(x) \equiv \varepsilon w^{\prime}(x)+a(x) w(x)=0, \quad x>0, \quad \ell w=-\ell v
$$

correspondingly. In view of $[9$, p. 13]

$$
|v(x)| \leq \frac{1}{a} \max _{0 \leq t \leq X}|L v(t)|, \quad 0 \leq x \leq X .
$$

Therefore, the estimate

$$
|w(x)| \leq \tilde{c} \max _{0 \leq t \leq X}|L v(t)|, \quad 0 \leq x \leq X
$$

holds with the corresponding constant $\tilde{c}$ independent on $\varepsilon$ and $x$ (see Theorem 2.5, Corollary 2.6, Theorem 3.3, Corollary 3.7). Taking into account the boundedness of $g(x)$ we finally get the desired estimate $\left|R_{\varepsilon, M}(x)\right| \leq C \varepsilon^{M+1}$, $0 \leq x \leq X$ with the constant $C$ independent on $\varepsilon$ and $x$. Theorem 5.1 is proved.

Note that the proof of Theorem 5.1 is extendable to NLIVP with integral kind of NLIVC considered in Section 4, so that for such NLIVP an appropriate asymptotic expansion of smooth solution holds.

Note that a possible initial layer overall appearing for smooth solution of NLIVP depends on $\phi$ of course as well as for the classical solution of a Cauchy problem. But for NLIVP the initial layer also depends on nonlocal factors as well as on the layout of nonlocal points. For example, we observe that the initial layer of the solution disappears for the problem $\varepsilon u^{\prime}(x)+u(x)=x, \quad x>0, u(0)-\alpha u(\zeta)=\phi$ with $\varepsilon>0$ and $\alpha=-\phi / \zeta$ for $\varepsilon \rightarrow 0$ and $\ell \omega(\varepsilon)=1-\alpha \exp (-\zeta / \varepsilon) \neq 0$. 


\section{Conclusion}

Finally, we emphasize, that the investigation of this paper is necessary for further research and construction of a uniform on small parameter finite-difference schemes for multipoint NLIVP. The author would like to express his gratitude to Prof. A. Ashyralyev for discussing author's investigation at the Institute of Mechanics and Mathematics of the Academy of Sciences of Turkmenistan which stimulated author's research.

\section{Bibliography}

[1] A. N. Tikhonov, On the dependence of the solutions of differential equations on a small parameter, Mat. Sb. 22(64) (1948), 193-204.

[2] M. I. Vishik and L. A. Lyusternik, Regular degeneration and boundary layer for linear differential equations with small parameter, Uspekhi Mat. Nauk 12:5(77) (1957), 3-122.

[3] A. V. Bitsadze and A. A. Samarskii, Some elementary generalizations of linear elliptic boundary value problems, Dokl. Akad. Nauk SSSR 185(4) (1969), 739-740.

[4] V. A. Il'in and E. I. Moiseev, Fist kind nonlocal boundary value problem for SturmLiouville operator in differential and difference treatment, Differential Equations 23(7) (1987), 1198-1207.

[5] V. A. Il'in and E. I. Moiseev, Second kind nonlocal boundary value problem for Sturm-Liouville operator, Differential Equations 23(8) (1987), 1422-1431.

[6] V. A. Il'in and E. I. Moiseev, 2-d nonlocal boundary-value problem for Poisson's operator in differential and difference variants, Mathematical Modelling 2(8) (1990), 130-156.

[7] A. B. Vasil'eva and B. F. Butuzov, Asymptotic Methods in the Theory of Singular Perturbations, Moscow, 1990.

[8] A. Ashyralyev and P. E. Sobolevskii, New Difference Schemes for Partial Differential Equations, Vol. 148, Birkhäuser, Basel, 2004.

[9] E. P. Doolan, J. J. H. Miller and W. H. A. Schilders, Uniform Numerical Methods for Problems with Initial and Boundary Layers, Boole Press, Dublin, 1980.

[10] A. S. Erdogan and S. N. Tekalan, First-order partial differential equation with a nonlocal boundary condition, Numerical Functional Analysis and Optimization 38(10) (2017), 1373-1389.

[11] M. A. Sadybekov, Stable difference scheme for a nonlocal boundary value heat conduction problem, e-Journal of Analysis and Applied Mathematics 2018(1) (2018), 1-10. 
[12] C. Ashyralyyev, Well-posedness of boundary value problems for reverse parabolic equation with integral condition, e-Journal of Analysis and Applied Mathematics 2018(1) (2018), 11-21.

[13] D. M. Dovletov, On the nonlocal boundary value problem of the first kind in differential and difference interpretation, Differential Equations 25(8) (1989), 917-924.

[14] D. M. Dovletov, Nonlocal boundary value problem in terms of flow for SturmLiouville operator in differential and difference statements, e-Journal of Analysis and Applied Mathematics 2018(1) (2018), 37-55.

[15] D. M. Dovletov, Differential and difference variants of 2-d nonlocal boundary value problem with Poisson's operator, AIP Conf. Proc. 2183 (2019), 070021, DOI: $10.1063 / 1.5136183$.

Received September 2, 2019; revised December 2, 2019; accepted December 9, 2019.

\section{Author information}

Dovlet M. Dovletov, Department of Mathematics, Near East University, Nicosia, TRNC, Mersin 10, Turkey.

E-mail: dovlet.dovletov@gmail .com 